\title{
Qualidade de vida relacionada à saúde de gestantes e fatores associados
}

\author{
Health-related quality of life of pregnant women and associated factors \\ Calidad de vida relacionada con la salud de gestantes y factores asociados \\ Paula Renata Amorim Lessa Soares ${ }^{1}$ in https://orcid.org/0000-0003-1629-443X \\ Cinthia Gondim Pereira Calou ${ }^{2}$ ic nttps://orcid.org/0000-0003-3488-6965 \\ Eveliny Silva Martins ${ }^{1}$ @i https://orcid.org/0000-0002-0013-9470 \\ Gilmara de Lucena Beserra ${ }^{1}$ i https://orcid.org/0000-0002-1195-2264 \\ Isael Cavalcante Silva ${ }^{1}$ io https:/orcid.org/0000-0002-1648-6522 \\ Samila Gomes Ribeiro ${ }^{1}$ ic hitps://orcid.org/0000-0002-4775-5852 \\ Priscila de Souza Aquino ${ }^{1}$ id hitps://orcid.org/0000-0003-4976-9817 \\ Ana Karina Bezerra Pinheiro' io https://orcid. org/0000-0003-3837-4131
}

Soares PR, Calou CG, Como citar: Silva IC, Ribeiro SG, et al. Qualidade de vida relacionada à saúde de gestantes e fatores associados. Acta Paul Enferm. 2021;34:eAPE002075.

DOI

http://dx.doi.org/10.37689/actaape/2021A0002075

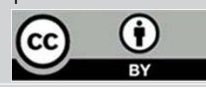

Qualidade de vida; Gravidez; Cur Fatores de risco; Saúde da mulher

Keywords

Quality of life; Pregnancy; Prenatal care; Risk factors; Women's health

Descriptores

Calidad de vida; Embarazo; Atención prenatal: Fatores de riesgo; Salud de la Mujer

Submetido

3 de Agosto de 2020

Aceito

3 de Dezembro de 2020

Autor correspondente

Paula Renata Amorim Lessa Soares Email: paularenata|@yahoo.com.br

\section{Resumo}

Objetivo: Identificar a influência dos fatores sociodemográficos, obstétricos e comportamentais na qualidade de vida de gestantes.

Métodos: Trata-se de um estudo transversal, correlacional, realizado entre os meses de setembro a janeiro de 2015, em quatro locais distintos de saúde: três unidades básicas de saúde e um serviço privado de imagem obstétrica e ginecológica. A amostra foi composta por 261 gestantes de baixo risco. Utilizou-se questionário socioeconômico, obstétrico e comportamental e o Índice de Qualidade de Vida de Ferrans \& Powers adaptado para mensuração da qualidade de vida de gestantes.

Resultados: Os fatores sociodemográficos tiveram associação estatisticamente significativa com a maior idade e escolaridade, maior renda, gestantes com parceiro estável e que tinham trabalho remunerado, revelando que essas mulheres possuem melhor qualidade de vida. No que tange aos dados obstétricos, gestantes com história de parto abdominal expressaram melhor qualidade de vida. Ademais, mulheres que tinham um ou mais filhos apresentaram pior qualidade de vida. Já quanto aos dados comportamentais gestantes com apoio do parceiro, que planejaram sua gestação, que receberam orientações educativas e que praticavam atividade física e que foram acompanhadas no serviço privado durante a gestação, apresentaram melhores índices de qualidade de vida.

Conclusão: Alguns fatores sociodemográficos, obstétricos e comportamentais possuem influência direta na qualidade de vida de gestantes, devendo ser priorizados no atendimento pré-natal.

\section{Abstract}

Objective: To identify the influence of sociodemographic, obstetric and behavioral factors on the quality of life of pregnant women.

Methods: This is a cross-sectional, correlational study conducted between September and January 2015 in four different health locations: three basic health centers and a private obstetric and gynecological imaging service. The sample consisted of 261 low-risk pregnant women. A socioeconomic, obstetric and behavioral questionnaire and the adapted Ferrans \& Powers Quality of Life Index were used to measure the quality of life of pregnant women.

Results: Sociodemographic factors had a statistically significant association with older age and schooling, higher income, pregnant women with a steady partner and paid work, revealing that these women have better quality of life. Regarding obstetric data, pregnant women with a history of abdominal delivery expressed a better quality of life. Women who had one or more children had worse quality of life. As for behavioral data, pregnant women who had partner's support, planned their pregnancy, received educational guidance, practiced physical activity and were in follow-up in the private service during pregnancy had better quality of life indexes. 
Conclusion: Some sociodemographic, obstetric and behavioral factors directly influence the quality of life of pregnant women and should be prioritized in prenatal care.

\section{Resumen}

Objetivo: Identificar la influencia de los factores sociodemográficos, obstétricos y de comportamiento en la calidad de vida de mujeres embarazadas.

Métodos: Se trata de un estudio transversal correlacional, realizado entre los meses de septiembre y enero de 2015, en cuatro lugares diferentes de salud: tres unidades básicas de salud y un servicio privado de imágenes obstétricas y ginecológicas. La muestra estuvo compuesta por 261 gestantes de bajo riesgo. Se utilizó un cuestionario socioeconómico, obstétrico y de comportamiento y el Índice de Calidad de Vida de Ferrans y Powers adaptado para medir la calidad de vida de mujeres embarazadas.

Resultados: Los factores sociodemográficos tuvieron una asociación estadísticamente significativa con mayor edad y escolaridad, mayores ingresos, gestantes con pareja estable y que tenían trabajo asalariado, lo que reveló que estas mujeres tienen una mejor calidad de vida. En lo que atañe a los datos obstétricos, gestantes con historia de parto abdominal expresaron una calidad de vida mejor. Además, mujeres que tenían un hijo o más presentaron peor calidad de vida. Con relación a los datos de comportamiento, las mujeres embarazadas con apoyo de su pareja, que planificaron la gestación, recibieron instrucciones educativas, practicaban actividad física y fueron tratadas en el servicio privado durante el embarazo presentaron mejores índices de calidad de vida.

Conclusión: Algunos factores sociodemográficos, obstétricos y de comportamiento tienen una influencia directa en la calidad de vida de gestantes y deben ser priorizados en la atención prenatal.

\section{Introdução}

A gestação caracteriza-se como um momento marcado por intensas transformaçóes na vida da mulher, sejam elas físicas, psicológicas, pessoais, emocionais, econômicas e sociais. ${ }^{(1)}$ Apesar de ser um processo fisiológico, a gravidez pode afetar intensamente, de forma negativa, a vida das mulheres, causando impactos direto na qualidade de vida $(\mathrm{QV})$ destas.

A QV configura-se como um construto subjetivo, multidimensional e possui diversos conceitos. Segundo a Organização Mundial da Saúde (OMS), a QV pode ser definida como a percepçáo do indivíduo quanto à sua posição na vida, levando em consideração o contexto cultural e valores nos quais ele vive em relação às suas expectativas de vida, objetivos pessoais, padróes e preocupações. ${ }^{(2)}$

Desse modo, a QV perpassa como foco de estudo de diversos campos de conhecimento, haja vista seu aspecto transversal. Todavia, na área da saúde denomina-se qualidade de vida relacionada à saúde (QVRS) e comporta-se como a importância que o indivíduo destina à sua saúde, tornando-se essencial para a investigaçáo em enfermagem, cujo sujeito principal do cuidado está voltado para a pessoa/família na sua integralidade. ${ }^{(3)}$

Assim, por ser algo pessoal e subjetivo a QVRS de gestantes pode variar intensamente, a depender da forma que a mulher percebe a sua vida e de acordo com o que ela considera importante. Sendo assim, fatores sociodemográficos, obstétricos e comportamentais podem influenciar diretamente a QVRS das gestantes.
A QVRS de gestantes é alvo de estudo entre pesquisadores, contudo, geralmente está associada a uma disfunção, seja de forma física, — como a função sexual, incontinência urinária ou lombalgia ou seja de ordem psicológica, — como a depressáo e ansiedade. ${ }^{(4,5)}$ Ademais, não há consenso quando se investiga quais os fatores que podem afetar esse construto.

Corroborando com tal afirmação, estudo realizado com o objetivo de identificar fatores associados à baixa QV de gestantes de alto risco evidenciou que dentre todas as variáveis sociodemográficas e obstétricas associadas, a única variável associada à baixa QV foi a ausência do parceiro. ${ }^{(6)}$

Isto posto, o presente artigo tem como objetivo identificar a influência dos fatores sociodemográficos, obstétricos e comportamentais na QV de gestantes.

\section{Métodos}

Trata-se de um estudo transversal, correlacional realizado em quatro locais distintos de saúde: três unidades básicas de saúde que atendem gestantes pelo sistema de saúde público e um serviço de imagem obstétrica e ginecológica associado ao sistema de saúde particular.

A população foi composta por gestantes de risco habitual e os critérios de inclusão foram grávidas em acompanhamento pré-natal de baixo risco, uma vez que as complicaçóes podem interferir na $\mathrm{QV}$ 
das gestantes; e alfabetizadas, devido ao preenchimento autoaplicável do instrumento do Índice de Qualidade de Vida Ferrans \& Powers adaptado. $^{(7)}$

O tamanho da amostra foi calculado baseado no atendimento mensal às gestantes nos quatro locais de pesquisa, perfazendo aproximadamente $800 \mathrm{mu}$ lheres, número encontrado a partir do somatório de 500 mulheres atendidas mensalmente na Clínica Feminimagem e 298 gestantes em acompanhamento pré-natal nos outros três locais públicos, totalizando 798 gestantes. Assim, utilizando a fórmula para populaçóes finitas em que se considerou o $\mathrm{N}$ de 800 , os valores de $95 \%$ para o nível de confiança, de $5 \%$ para o erro máximo permitido, $50 \%$ para a porcentagem complementar e também de 50\% para a qual o fenômeno se verifica, a amostra totalizou em 261 gestantes, sendo 141 pertencentes ao sistema de saúde público e 120 ao serviço privado.

Para a coleta de dados utilizou-se um questionário composto por três partes: Parte I - dados sócio demográficos: idade, estado civil, escolaridade, raça, estado marital, ocupação, renda familiar e religião; Parte II - dados obstétricos: Índice de Massa Corporal (IMC), trimestre gestacional, início do acompanhamento pré-natal, paridade e tipo de parto. Parte III - Fatores comportamentais: gravidez planejada, recebimento de orientaçóes educativas na gestação, prática de atividade física, uso de cigarro, bebida alcoólica e drogas ilícitas.

Também utilizou-se um questionário para a mensuração da QV de gestantes, o Índice de Qualidade de Vida de Ferrans \& Powers adaptado que possui quatro domínios: "Saúde/funcionamento", "Psicológico/espiritual", "Socioeconômico" e "Família", com pontuação do valor total da escala e dos domínios variando de 0 a 30, sem ponto de corte, com os maiores valores indicando melhor QV.

A coleta de dados ocorreu entre os meses de setembro de 2014 a janeiro de 2015 e as participantes foram abordadas enquanto aguardavam atendimento, seja a consulta de pré-natal no serviço público ou a realização do exame de imagem obstétrico na assistência privada de saúde. Somente após aceite as gestantes eram encaminhadas, sem acompanhante, para uma sala reservada, garantindo o sigilo das informaçóes fornecidas.
Os dados sócio demográficos, obstétricos e comportamentais foram compilados e analisados por meio do programa estatístico Statistical Package for the Social Sciences (SPSS), versão 20.0. Calcularam-se médias e desvios-padrão das variáveis quantitativas. As associaçóes entre as variáveis foram feitas pelo teste de qui-quadrado, sendo consideradas estatisticamente significantes quando $\mathrm{p}<0,05$.

A pesquisa foi avaliada pelo Comitê de Ética e Pesquisa (CEP) da MEAC/UFC, sendo aprovado com o parecer número 770.902 .

\section{Resultados}

Correspondendo à avaliação da QV de gestantes, encontrou-se os seguintes resultados dispostos na tabela 1 .

Tabela 1. Comparação das médias da escala total que avalia a qualidade de vida a partir do instrumento de Ferrans \& Powers adaptado

\begin{tabular}{lc}
\hline Domínio & Média \pm DP \\
\hline Sáude/Funcionamento & $22,0 \pm 3,7^{\star}$ \\
Sócio econômico & $22,8 \pm 4,8^{\star}$ \\
Psicológico/Espiritual & $25,7 \pm 4,2^{\star}$ \\
Família & $27,4 \pm 3,4^{\star}$ \\
Total & $23,6 \pm 3,3^{\star}$ \\
\hline
\end{tabular}

*0 $p$-value de Friedman foi $<0,0001$. Pelo teste de Conover todas as médias diferem entre $s i(p<0,004)$

O escore total da escala evidenciou uma média de 23,6 com desvio padrão de 3,3, com maior pontuação no domínio "Família" $(27,4)$ e menor pontuação no domínio "Saúde/Funcionamento" $(22,0)$. Os resultados também apontaram que todas as médias diferiram entre si $(\mathrm{p}<0,004)$. A tabela 2 versa sobre a associação das variáveis sociodemográficas com o índice de QV de Ferrans \& Powers adaptado.

Todas as variáveis analisadas revelaram associação significativa com, no mínimo, dois domínios da escala e com o domínio total da escala, com exceção do estado marital. Destaca-se a variável religião, que evidenciou associação estatística com todos os domínios da escala. A tabela 3 reporta sobre a associação das variáveis obstétricas com o índice de QV de Ferrans \& Powers adaptado.

A associação das variáveis obstétricas evidenciou associação significativa com o tipo de parto e o nú- 
Tabela 2. Associação das variáveis sociodemográficas com o índice de qualidade de vida de Ferrans \& Powers

\begin{tabular}{|c|c|c|c|c|c|}
\hline Variáveis sociodemográficas & $\begin{array}{l}\text { D1- Saúde } \\
\text { Média } \pm \text { DP }\end{array}$ & $\begin{array}{c}\text { D2- Socioeconômico } \\
\text { Média } \pm \text { DP }\end{array}$ & $\begin{array}{l}\text { D3- Psicológico } \\
\text { Média } \pm \text { DP }\end{array}$ & $\begin{array}{l}\text { D4- Família } \\
\text { Média } \pm \text { DP }\end{array}$ & $\begin{array}{c}\text { D- Total } \\
\text { Média } \pm \text { DP }\end{array}$ \\
\hline \multicolumn{6}{|l|}{ Idade (ano) } \\
\hline$<20$ & $21,5 \pm 4,0$ & $19,5 \pm 5,3$ & $25,8 \pm 4,0$ & $26,8 \pm 4,7$ & $22,5 \pm 3,8$ \\
\hline Entre 21 e 30 & $22,19 \pm 3,6$ & $22,96 \pm 4,7$ & $25,5 \pm 4,5$ & $27,5 \pm 3,1$ & $23,6 \pm 3,4$ \\
\hline$>31$ & $22,05 \pm 3,7$ & $24,38 \pm 3,8$ & $25,9 \pm 3,9$ & $27,7 \pm 2,7$ & $24,0 \pm 3,0$ \\
\hline$p$-value & 0,639 & 0,000 & 0,763 & 0,317 & 0,045 \\
\hline \multicolumn{6}{|l|}{ Estado marital } \\
\hline Sem parceiro & $21,8 \pm 3,7$ & $20,3 \pm 5,9$ & $25,5 \pm 5,6$ & $25,8 \pm 5,2$ & $22,9 \pm 3,8$ \\
\hline Com parceiro & $22,0 \pm 3,7$ & $23,1 \pm 4,6$ & $25,7 \pm 4,0$ & $27,6 \pm 3,0$ & $23,6 \pm 3,3$ \\
\hline$p$-value & 0,827 & 0,003 & 0,777 & 0,008 & 0,297 \\
\hline \multicolumn{6}{|l|}{ Escolaridade } \\
\hline 8 anos ou menos & $21,5 \pm 4,8$ & $19,4 \pm 5,9$ & $24,2 \pm 6,8$ & $26,5 \pm 4,3$ & $22,3 \pm 4,6$ \\
\hline 9 a 11 anos & $21,9 \pm 4,0$ & $21,7 \pm 5,3$ & $25,7 \pm 4,6$ & $27,4 \pm 4,6$ & $23,2 \pm 3,8$ \\
\hline 12 anos completos & $21,6 \pm 3,5$ & $21,9 \pm 4,8$ & $25,6 \pm 4,0$ & $27,3 \pm 3,0$ & $23,1 \pm 3,1$ \\
\hline Acima de 13 anos & $22,4 \pm 3,3$ & $24,7 \pm 3,3$ & $26,3 \pm 2,8$ & $27,8 \pm 2,6$ & $24,3 \pm 2,6$ \\
\hline$p$-value & 0,408 & 0,000 & 0,072 & 0,284 & 0,007 \\
\hline \multicolumn{6}{|l|}{ Trabalha fora } \\
\hline $\operatorname{Sim}$ & $22,4 \pm 3,3$ & $24,5 \pm 3,5$ & $25,9 \quad 3,7$ & $27,6 \pm 3,1$ & $24,2 \pm 207$ \\
\hline Não & $21,4 \pm 4,2$ & $20,3 \pm 5,4$ & $25,5 \pm 4,7$ & $27,2 \pm 3,7$ & $22,7 \pm 3,9$ \\
\hline$p$-value & 0,046 & 0,000 & 0,435 & 0,409 & 0,000 \\
\hline \multicolumn{6}{|l|}{ Renda } \\
\hline De $1 \mathrm{a} \leq 2$ & $21,6 \pm 4,2$ & $20,2 \pm 5,3$ & $24,7 \pm 5,5$ & $26,6 \pm 4,3$ & $22,6 \pm 4,4$ \\
\hline$>2 \mathrm{a} \leq 4$ & $21,7 \pm 3,8$ & $23,0 \pm 5,1$ & $26,0 \pm 4,1$ & $27,9 \pm 3,0$ & $23,6 \pm 3,3$ \\
\hline$>4 \mathrm{a} \leq 6$ & $22,3 \pm 3,3$ & $23,7 \pm 3,9$ & $27,6 \pm 1,8$ & $28,3 \pm 2,0$ & $24,4 \pm 2,6$ \\
\hline Mais de 6 & $22,4 \pm 3,4$ & $24,9 \pm 3,2$ & $26,1 \pm 2,8$ & $27,7 \pm 2,7$ & $24,3 \pm 2,6$ \\
\hline$p$-value & 0,490 & 0,000 & 0,011 & 0,034 & 0,004 \\
\hline \multicolumn{6}{|l|}{ Religião } \\
\hline Católica & $22,6 \pm 3,2$ & $23,8 \pm 4,1$ & $26,2 \pm 3,5$ & $27,9 \pm 2,7$ & $24,2 \pm 2,8$ \\
\hline Evangélica & $21,3 \pm 3,9$ & $21,6 \pm 5,4$ & $25,8 \pm 4,2$ & $27,4 \pm 4,0$ & $23,0 \pm 3,5$ \\
\hline Nenhuma & $21,4 \pm 5,9$ & $20,8 \pm 6,5$ & $24,5 \pm 7,1$ & $25,8 \pm 4,0$ & $22,4 \pm 5,6$ \\
\hline$p$-value & 0,024 & 0,002 & 0,001 & 0,003 & 0,003 \\
\hline
\end{tabular}

Tabela 3. Associação das variáveis obstétricas com o índice de qualidade de vida de Ferrans \& Powers

\begin{tabular}{|c|c|c|c|c|c|}
\hline Variáveis obstétricas & D1- Saúde* & D2- Socioeconômico* & D3- Psicológico* & D4- Família* & D- Total $^{\star}$ \\
\hline \multicolumn{6}{|l|}{ Idade gestacional } \\
\hline $1^{0}$ trimestre & $21,4 \pm 4,2$ & $22,5 \pm 5,3$ & $25,5 \pm 4,3$ & $27,5 \pm 3,0$ & $23,2 \pm 3,7$ \\
\hline $2^{0}$ trimestre & $22,2 \pm 3,4$ & $22,9 \pm 4,7$ & $25,4 \pm 4,5$ & $27,2 \pm 3,4$ & $23,7 \pm 3,1$ \\
\hline $3^{0}$ trimestre & $22,0 \pm 3,9$ & $22,9 \pm 4,8$ & $26,2 \pm 3,6$ & $27,7 \pm 3,5$ & $23,6 \pm 3,4$ \\
\hline$p$-value & 0,469 & 0,887 & 0,433 & 0,497 & 0,691 \\
\hline \multicolumn{6}{|l|}{ IMC gestacional } \\
\hline Baixo peso & $21,9 \pm 3,5$ & $22,3 \pm 4,3$ & $25,7 \pm 3,7$ & $27,4 \pm 3,1$ & $23,4 \pm 2,9$ \\
\hline Adequado & $22,1 \pm 3,6$ & $22,8 \pm 4,7$ & $25,8 \pm 4,0$ & $27,4 \pm 3,3$ & $23,7 \pm 3,2$ \\
\hline Sobrepeso & $21,9 \pm 4,0$ & $22,6 \pm 5,2$ & $25,3 \pm 4,8$ & $27,4 \pm 3,1$ & $23,4 \pm 3,7$ \\
\hline Obesidade & $21,3 \pm 3,9$ & $23,4 \pm 5,3$ & $25,6 \pm 4,2$ & $27,5 \pm 4,4$ & $23,4 \pm 3,6$ \\
\hline$p$-value & 0,758 & 0,839 & 0,899 & 0,998 & 0,916 \\
\hline \multicolumn{6}{|l|}{ Tipo de parto ( $\mathrm{n}=113$ ) } \\
\hline Vaginal & $21,1 \pm 5,2$ & $21,5 \pm 5,4$ & $23,1 \pm 6,6$ & $26,3 \pm 3,4$ & $22,3 \pm 4,6$ \\
\hline Abdominal & $21,7 \pm 3,5$ & $23,8 \pm 4,3$ & $25,7 \pm 3,6$ & $27,8 \pm 3,2$ & $23,7 \pm 3,0$ \\
\hline$p$-value & 0,513 & 0,017 & 0,008 & 0,026 & 0,072 \\
\hline \multicolumn{6}{|l|}{$N^{0}$ de filhos vivos } \\
\hline 0 & $22,2 \pm 3,4$ & $22,6 \pm 4,8$ & $26,2 \pm 3,6$ & $27,5 \pm 3,4$ & $23,7 \pm 3,1$ \\
\hline $1-7$ & $21,6 \pm 4,1$ & $23,1 \pm 4,9$ & $25,0 \pm 4,9$ & $27,4 \pm 3,4$ & $23,3 \pm 3,6$ \\
\hline$p$-value & 0,181 & 0,367 & 0,032 & 0,799 & 0,408 \\
\hline
\end{tabular}

${ }^{*}$ Média \pm Desvio Padrão (DP)

mero de filhos. Essa variável mostrou significância com o domínio "Psicológico/espiritual", em que nulíparas apresentaram melhor QV. As mulheres que já vivenciaram gestação anterior apresentaram índices maiores de QV em todos os domínios, as que se submeteram ao parto abdominal em com- 
Tabela 4. Associação dos fatores comportamentais com o índice de qualidade de vida de Ferrans \& Powers

\begin{tabular}{|c|c|c|c|c|c|}
\hline Fatores relacionado a QVRS & $\begin{array}{l}\text { D1- Saúde } \\
\text { Média } \pm \text { DP }\end{array}$ & $\begin{array}{c}\text { D2- Socioeconômico } \\
\text { Média } \pm \text { DP }\end{array}$ & $\begin{array}{c}\text { D3- Psicológico } \\
\text { Média } \pm \text { DP }\end{array}$ & $\begin{array}{l}\text { D4- Família } \\
\text { Média } \pm \text { DP }\end{array}$ & $\begin{array}{c}\text { D- Total } \\
\text { Média } \pm \text { DP }\end{array}$ \\
\hline \multicolumn{6}{|l|}{ Gravidez Planejada } \\
\hline $\operatorname{Sim}$ & $22,3 \pm 3,1$ & $24,0 \pm 3,7$ & $26,4 \pm 2,9$ & $27,8 \pm 2,9$ & $24,1 \pm 2,5$ \\
\hline Não & $21,6 \pm 4,3$ & $21,4 \pm 5,6$ & $24,9 \pm 5,2$ & $27,0 \pm 3,8$ & $22,9 \pm 4,0$ \\
\hline$p$-value & 0,110 & 0,000 & 0,005 & 0,054 & 0,003 \\
\hline \multicolumn{6}{|l|}{ Apoio do parceiro } \\
\hline $\operatorname{Sim}$ & $22,1 \pm 3,6$ & $23,0 \pm 4,7$ & $25,8 \pm 3,9$ & $27,6 \pm 3,3$ & $23,7 \pm 3,2$ \\
\hline Não & $19,8 \pm 4,7$ & $18,2 \pm 5,7$ & $22,8 \pm 7,6$ & $24,6 \pm 4,1$ & $21,0 \pm 4,3$ \\
\hline$p$-value & 0,038 & 0,001 & 0,016 & 0,003 & 0,010 \\
\hline \multicolumn{6}{|l|}{ Educação em saúde } \\
\hline $\mathrm{Sim}$ & $22,8 \pm 3,3$ & $23,5 \pm 4,4$ & $26,4 \pm 3,6$ & $27,6 \pm 3,1$ & $24,3 \pm 2,9$ \\
\hline Não & $21,4 \pm 3,9$ & $22,4 \pm 5,1$ & $25,2 \pm 4,5$ & $27,3 \pm 3,5$ & $23,1 \pm 3,5$ \\
\hline$p$-value & 0,002 & 0,070 & 0,19 & 0,430 & 0,005 \\
\hline \multicolumn{6}{|l|}{ Atividade física } \\
\hline $\operatorname{Sim}$ & $23,5 \pm 3,0$ & $24,2 \pm 4,0$ & $25,8 \pm 2,9$ & $28,0 \pm 2,3$ & $24,6 \pm 2,6$ \\
\hline Não & $21,7 \pm 3,8$ & $22,6 \pm 4,9$ & $25,7 \pm 4,3$ & $27,3 \pm 3,5$ & $23,4 \pm 3,4$ \\
\hline$p$-value & 0,008 & 0,079 & 0,854 & 0,275 & 0,041 \\
\hline \multicolumn{6}{|l|}{ Tipo de serviço } \\
\hline Público & $21,6 \pm 4,0$ & $21,1 \pm 5,3$ & $25,1 \pm 5,0$ & $27,0 \pm 3,9$ & $22,8 \pm 3,7$ \\
\hline Privado & $22,5 \pm 3,3$ & $24,9 \pm 3,3$ & $26,4 \pm 2,7$ & $27,9 \pm 2,5$ & $24,4 \pm 2,6$ \\
\hline$p$-value & 0,051 & 0,000 & 0,013 & 0,028 & 0,000 \\
\hline
\end{tabular}

paração com as que tiveram parto vaginal, tendo associação estatisticamente significativa nos domínios "Socioeconômico", "Psicológico" e "Família". A tabela 4 traz a influência dos aspectos comportamentais na QV de gestantes.

Os dados demonstraram associação significativa de todas as variáveis avaliadas com o domínio total da escala. Assim, o planejamento da gestação, o recebimento das orientaçóes educativas durante o pré-natal, a prática de atividade física e gestantes que foram atendidas no serviço privado possuem melhor QVRS.

\section{Discussão}

A presente pesquisa apresentou como limitação o desenho metodológico transversal que diminui o poder de inferência de causa e efeito entre as variáveis associadas.

Todavia, torna-se imprescindível conhecer os fatores sociodemográficos, obstétricos e comportamentais que podem comprometer a QV das gestantes, no sentido de reconhecer as vulnerabilidades e direcionar a assistência com vistas a minimizar os impactos negativos e proporcionar melhoria na QV nesse período de intensas transformaçóes na vida da mulher.
Foi evidenciada média da escala total de 23,6, considerada satisfatória, com maior pontuação nos domínios "Família" $(27,4)$ e "Psicológico/espiritual" $(25,7)$. Em consonância com esse achado, pesquisa realizada com 250 mulheres, que teve como escopo mensurar a QVRS de gestantes, encontrou média da escala total de 23,8 e o domínio com maior pontuação também foi "Família" $(27,22) .{ }^{(7)}$

A associação entre as variáveis sociodemográficas com o índice de QVRS de gestantes apontou para importantes achados, revelando que a idade e a escolaridade foram significantes no domínio "Socioeconômico" e na escala total, com diferenças maiores entre os extremos das categorias.

Sabe-se que a idade mais avançada em gestantes pode interferir de forma positiva na QVRS, uma vez que devido às diversas experiências vivenciadas, essas são capazes de desenvolver métodos de enfrentamento das adversidades mais eficazes. ${ }^{\left({ }^{(8)}\right.}$

A situação conjugal obteve relação de influência com os domínios "Socioeconômico" e "Familiar". A presença do parceiro é fonte de apoio e segurança para a gestante, sendo capaz de elevar sua autoestima durante a gravidez e melhorar a percepçáo destas quanto à sua QVRS. Acrescenta-se ainda a importância do parceiro e do seu apoio emocional na construção da identidade da maternidade. ${ }^{(6)}$ 
Estudo que avaliou a qualidade de vida de 552 mães na Coreia do Sul também demonstrou dados semelhantes, no qual as mães solteiras têm menor QV do que as casadas. Além de serem mais propensas a ter níveis mais elevados de estresse e sintomas de depressão. ${ }^{(9)}$

No presente estudo, as mulheres que tinham ocupação remunerada e com maior renda familiar tiveram maior QVRS em quase todos os domínios e na escala total, demonstrando, talvez, que a segurança de possuir um trabalho e, consequentemente, um maior aporte financeiro pode ser preponderante para sua satisfação e manutenção de sua saúde do que os desgastes provocados pelo trabalho.

Mulheres que possuíam uma religião apresentam melhores índices de QVRS em todas as nuances pesquisadas: saúde, socioeconômico, psicológico e familiar. A religião possui forte influência sobre a $\mathrm{QV}$ em diferentes fases da vida, tornando-se relevante para promover saúde e prevenção de agravos à população..$^{(10)}$

No que tange às variáveis obstétricas, os dados mostraram que mulheres que tiveram parto abdominal apresentaram índices maiores de QVRS, em todos os domínios, do que mulheres que tiveram parto vaginal, com significância nos domínios "Socioeconômico", "Psicológico" e "Família".

Pesquisas divergem quanto à influência do tipo de parto na QVRS das mulheres. O Brasil caracteriza-se como um país marcado pelo alto número de cirurgias cesarianas. ${ }^{(1)}$ Todavia, inúmeros são os benefícios do parto vaginal para a mãe e para o bebê, minimizando os riscos para a saúde de ambos, ${ }^{(12)} \mathrm{o}$ que, consequentemente, melhora a QV no pós-parto das mulheres, principalmente se este for realizado sem episiotomia.

Ao investigar mulheres que deram à luz de maneiras diferentes, foi evidenciado diferenças significativas entre a $\mathrm{QV}$ das mulheres com índices crescentes entre as que tiveram parto vaginal sem episiotomia, seguido das que tiveram parto vaginal com episiotomia e com pior índice aquelas que deram à luz por cesariana. Logo, a adoção de boas práticas na assistência ao parto pode influencar na QV das mulheres. ${ }^{(13)}$

No que diz respeito ao número de filhos, mulheres que não tinham nenhum filho apresentaram melhores índices de QV do que mulheres que já os possuíam, com significância estatística no domínio "Psicológico/espiritual".

A chegada de outro filho no contexto familiar exige adaptaçóes de todos os membros, e pode contribuir para o aumento do estresse e privação do sono da mãe. Ademais, essa nova realidade pode levar a um afastamento do casal e de suas atividades pessoais, ${ }^{(14)}$ contribuindo para essa diminuição da QV no domínio "Psicológico/espiritual" encontrado na presente pesquisa.

Quanto aos fatores comportamentais relacionados à QVRS, o planejamento da gravidez e o apoio do parceiro mostrou associação em quase todos os domínios, indicando a importância de se programar o momento da maternidade e paternidade para que se tenha maior satisfação e melhor QVRS.

Estudo realizado no Nordeste brasileiro com 652 puérperas sugere que uma gravidez planejada contribui para maior satisfação e melhor QV da mulher, que, ao descobrir a gravidez, motiva-se, inclusive, para a realização de um pré-natal com melhores indicadores, como início precoce do acompanhamento. $^{(15)}$

A associação dos domínios de QVRS com alguns fatores também revelou que mulheres que verbalizaram terem recebido orientaçóes educativas durante o pré-natal, que praticavam atividade física e que foram atendidas no serviço privado tiveram maiores pontuaçóes e melhor QVRS com associação significante.

Pode-se presumir que o recebimento das orientaçóes educativas talvez tenha auxiliado as gestantes no enfrentamento dos sintomas físicos que a gestação traz, contribuindo para que essas mulheres assistidas no âmbito da educação em saúde tivessem melhor QVRS, como retratam os números do estudo.

Por fim, observou-se que mulheres atendidas no serviço público apresentaram piores índices de QVRS em todos os domínios, com exceção do domínio "Saúde" em comparação com gestantes do serviço privado, evidenciando, talvez, a relevância do aspecto socioeconômico na QVRS das mulheres. Contudo, deve-se aprofundar a investigação a fim de saber se outras variáveis, como renda, escolari- 
dade e rede de apoio, comportam-se como variáveis confundidoras.

\section{Conclusão}

As gestantes com maior idade, maior escolaridade, com parceiro estável, que trabalhavam fora, com alta renda familiar, que possuem religiaao, com história de parto abdominal, nulíparas, com gestação planejada, com apoio do parceiro, que receberam orientações educativas, que praticavam atividade física e que foram atendidas no serviço privado apresentaram melhores índices de qualidade de vida. Dessa forma, alguns fatores sociodemográficos, obstétricos e comportamentais causam influência direta na QV de gestantes.

\section{Colaborações}

Soares PRAL, Calou CGP, Martins ES, Beserra GL, Silva IC, Ribeiro SG, Aquino PS e Pinheiro AKB declaram que contribuíram com a concepção do estudo, análise e interpretação dos dados, redação do artigo, revisão crítica relevante do conteúdo intelectual e aprovação da versão final a ser publicada.

\section{Referências}

1. Lima MO, Tsunechiro MA, Bonadio IC, Murata M. Depressive symptoms in pregnancy and associated factors: Iongitudinal study. Acta Paul Enferm. 2017;30(1):39-46.
2. World Health Organization (WHO). Strategy for mental health and substance abuse in the Eastern Mediterranean Region 2012-2016. Geneva: WHO; 2011. [Technical paper; Regional Committee for the Eastern Mediterranean, Fifty-eighth Session].

3. Bica I, Pinho LM, Silva EM, Aparício G, Duarte J, Costa J, et al. Sociodemographic influence in health-related quality of life in adolescentes. Acta Paul Enferm. 2020;33:e-APE20190054.

4. Barros FL, Souza FR, Couto MD, Bezerra LL, Silva MB, Pacagnelli FL, et al. Effect of hydrotherapy treatment in lombar pain and quality of life of pregnant: case study. Colloq Vitae. 2018;10(1):74-9.

5. Ribas LL, Oliveira EA, Cirqueira RP, Ferreira JB. The impact of urinary incontinence on the quality of life of pregnant women. Rev Multidiscipl Psicol. 2019;13(43):431-9.

6. Trombeta JB, Traebert J, Nunes RD, Freschi LD. Factors associated with quality of life in high-risk pregnant. Arq Catarin Med. 2019;48(4):7587.

7. Ferrans CE, Powers MJ. Psychometric assessment of the Quality of Life Index. Res Nurs Health. 1992;15(1):29-38.

8. Barbosa AK, Queiroz BF, Teixeira DR, Mourão EC, Cantanhede GM, Carvalho KR, et al. Quality of life of low risk pregnant women in Teresina -PI. Rev Eletr Acervo Saúde. 2019;11(16):1-8.

9. Kim GE, Kim EJ. Factors affecting the quality of life of single mothers compared to married mothers. BMC Psychiatry. 2020;20(1):169.

10. Maffei B, Menezes M, Crepaldi MA. Significant social network in the gestational process: an integrative review. Rev SBPH. 2019;22(1):21637.

11. Fernandez JC, Silva RA, Sacardo DP. Religion and health: to transform absence in presence. Saude Soc. 2018;27(4):1058-70.

12. Gazineu RC, Amorim KR, Paz CT, Gramacho RC. Benefits of natural childbirth for the quality of life of the mother-child binomial. Textura. 2018;12(20):121-9.

13. Kohler S, Sidney Annerstedt K, Diwan V, Lindholm L, Randive B, Vora K, et al. Postpartum quality of life in Indian women after vaginal birth and cesarean section: a pilot study using the EQ-5D-5L descriptive system. BMC Pregnancy Childbirth. 2018;18(1):427.

14. Oliveira AC, Sene LB, Watanabe LA. Perception of pain in normal delivery in pregnant women. Scire Salutis. 2018;8(2):32-42.

15. Santos JM, Matos TS, Mendes RB, Freitas CK, Leite AM, Rodrigues ID. Influence in the reproductive planning and the women's satisfaction with the discovery of being pregnant in the quality of prenatal care in Brazil. Rev Bras Saúde Mater Infant. 2019;19(3):529-35. 